\title{
Die Aerzte Deutschlands im Jahre 1904.
} Von Dr. Georg Heimann in Berlin.

Im Jahre 1904 hat die Zahl der Aerzte im Deutschen Reiche etwas weniger als in den Vorjahren zugenommen. Sie betrug nach dem am 1. November 1904 abgeschlossenen Reichsmedizinalkalender, Jahrgang 1905, herausgegeben von Prof. Dr. Schwalbe, 30457 oder $460=1,6 \%$ mehr als Ende 1903 ; in den sieben vorhergehenden Jahren war die Zunahme $861,959,800,653,932,884,873$ gewesen. In Preußen wurden 18480 oder 261 mehr Aerzte als im Jahre 1903 gezählt, wo die Vermehrung 603 betragen hatte. In Bayern belief sich im Berichtsjahre die Aerztezahl auf $3299(+51)$; im Königreich Sachsen auf $2304(+91)$; in Württem berg auf $987(+10)$; im Großherzogtum Baden auf 1177 ( -7$)$; im Großherzogtum Hessen auf 731 $(-10)$; in Elsaß-Lothringen auf $871(+17)$. Baden und Hessen sind diejenigen größeren Bundesstaaten, welche, wie weiter unten gezeigt wird, im Verhältnis zur Einwohnerzahl die größte Aerztezahl haben. Dies macht die Abnahme, welche sich übrigens schon im Vorjahre für Baden feststellen ließ, leicht begreiflich. Es waren ferner Aerzte in

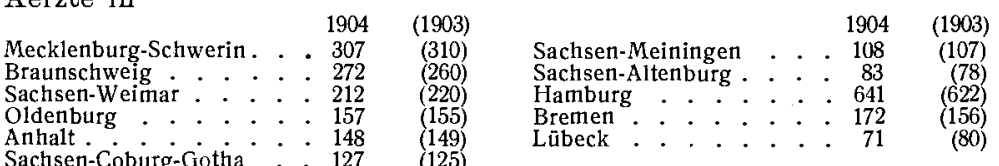

In Lippe-Detmold waren im Jahre 190454 (54), in Reuß j. L. 62 (60), in Schwarzburg-Rudolstadt 45 (43), in Waldeck 39 (35), in Mecklenburg-Strelitz 38 (36), in Schwarzburg-Sondershausen 36 (34), in Reuß ä. L. 19 (19) und in Schaumburg-Lippe 17 (18) Aerzte. Eine nennenswerte Veränderung ist demnach in den kleineren Staaten nicht eingetreten. Von den preußischen Provinzen hatte, wie früher, die Rheinprovinz die meisten, nämlich 2945 (2883) Aerzte. Es folgen, wenn wir von Berlin absehen:

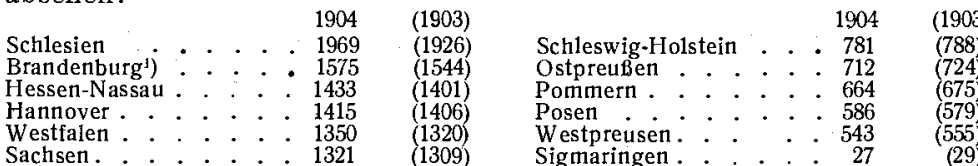

Im Vorjahre war für Schlesien eine Verminderung (um 11) zu beobachten gewesen. In diesem Jahre gilt dies besonders von den anderen östlichen Provinzen sowie Schleswig-Holstein und Pommern.

Von Einfluß auf die Verminderung der Zunahme der Aerztezahl im Deutschen Reiche während des Jahres $190 t$ ist die Einfülurung des praktischen Jahres gewesen, das, abgesehen von gewissen Ausnahmen in Berücksichtigung zwingender persönlicher Verhältnisse, alle diejenigen Kandidaten der Medizin vor Erteilung der Approbation abzuleisten haben, welche die ärztliche Prüfung vor dem 1. Oktober 1903 nicht vollständig bestanden hatten. Demzufolge ist in dem Berichts. jahr der Zugang von jungen Aerzten ein erheblich geringerer als in den Vorjahren gewesen, eine Erscheinung vorübergehender Natur, die vom nächsten Jahre ab nicht mehr in dem Maße zu beobachten sein wird. Ziffernmäßige Nachweisungen sind bisher amtlich nicht veröffentlicht, nur aus Bayern enthielten die Hochschulnachrichten eine Notiz, wonach die Zahl der Approbationen von 360 im Prüfungsjahr 1902/3 auf 295 im Prüfungsjahre 1903/4 zurückgegangen ist. In Preußen dürfte derRückgang verhältnismäßig erheblicher gewesen sein. Es wurden dort 1902/03 717, in den übrigen deutschen Staaten 474, zusammen im Deutschen Reiche 1551 ärztliche Approbationen, davon 12 an Aerztinnen, verliehen. Die Höhe dieser Zahlen ist wahrscheinlich auch durch das Bestreben der Kandidaten beeinflußt, vor dem 1. Oktober 1903 die Prüfung vollständig abzulegen, um noch ohne Absolvierung des praktischen Jahres die Approbation zu erhalten.

Wenn wir die Verteilung der Aerzte nach dem Flächenraum ins Auge fassen, so ergibt sich, daß im Deutschen Reiche auf $100 \mathrm{qkm}$ 5,63 Aerzte wohnten. Hoch ist die Ziffer für das Königreich Sachsen: 15,37; für das Großherzogtum Hessen beträgt sie 9,52; für Baden 7,80; für Elsaß-Lothringen 6,00; für Preußen 5,30; für Württemberg 5,06; für Bayern 4,35. Am ungünstigsten stehen, wie bisher, von den preußischen Regierungsbezirken da: Köslin 1,07; Gumbinnen 1,23; Marienwerder 1,41; von den bayerischen: Oberpfalz und Niederbayern mit 1,81,

1) Ohne Charlottenburg. 
beziehungsweise 1,85 Aerzten auf $100 \mathrm{qkm}$. Sehr hoch ist, wie auch früher, die Ziffer für den Regierungsbezirk Düsseldorf: 22,44.

A uf 10000 Einwohner kamen im Jahre 1904 im Deutschen Reiche 5,40; in Preußen 5,36; in Bayern 5,34; in Sachsen 5;48; in ElsaßLothringen 5,07 Aerzte; weniger in Württemberg: 4,55; mehr in den Großherzogtümern Baden und Hessen: 6,30, beziehungsweise 6,53. Für die Gebiete der Hansestädte Hamburg, Bremen, Lübeck waren die Ziffern beziehungsweise 8,34, 7,65, 7,34; für die kleineren Bundesstaaten bewegten sie sich zwischen 6,73 (Waldeck mit seinen Kurorten) und 2,78 (Reuß ä. L). Von den preußischen Regierungsbezirken hatte, wie in früheren Jahren, der an Kurorten reiche Bezirk Wiesbaden die höchste Zahl: 9,45; demnächst kamen die Bezirke Köln (8,13) und Aurich $(7,96)$; die niedrigste Marienwerder (2,76). Oppeln (2,74), Köslin $(2,55)$ und Gumbinnen $(2,47)$.

Was die Großstädte betrifft, so waren Aerzte in

\begin{tabular}{|c|c|c|c|c|c|}
\hline & & (1903) & & & \\
\hline Berlin & $\begin{array}{r}2638 \\
502\end{array}$ & (2572) & Aachen & 115 & $(114)$ \\
\hline $\begin{array}{l}\text { Brestau. } \\
\text { Kö́ln. }\end{array}$ & & (573) & $\begin{array}{l}\text { Essen } \\
\text { Posen }\end{array}$ & $\begin{array}{l}90 \\
139\end{array}$ & $\begin{array}{r}(99) \\
(141)\end{array}$ \\
\hline Frankfurt a. $\dot{M}$. & & (366) & Kiel. & 223 & $(235)$ \\
\hline Hannover. & 276 & & Crefeld & 69 & \\
\hline Magdeburg & 190 & (191) & Kassel . & 124 & (123) \\
\hline & & $(182)$ & Duisburg & $\begin{array}{r}49 \\
770\end{array}$ & (48) \\
\hline iigsberg & 1964 & (1891) & $\begin{array}{l}\text { Munchen } \\
\text { Dresden }\end{array}$ & & \\
\hline Altona. & 99 & (95) & Leipzig & 524 & (444) \\
\hline & & (88) & Stuttgart & & \\
\hline $\begin{array}{l}\text { le a.s. } \\
\text { tmund }\end{array}$ & $\begin{aligned} 209 \\
98\end{aligned}$ & $\begin{array}{l}(205) \\
(89)\end{array}$ & $\begin{array}{l}\text { Stráburg i. E. } \\
\text { Nürnberg. }\end{array}$ & $\begin{array}{l}248 \\
198\end{array}$ & (183) \\
\hline & 150 & (77) & Chemnitz & 132 & (130) \\
\hline & & & Braunschweig & & \\
\hline
\end{tabular}

In Groß-Berlin wohnten 3814 Aerzte gegen 3726 , beziehungsweise 3524 in den beiden Vorjahren. In der Reichshauptstadt selbst ist die Zunahme nicht gerade erheblich gewesen: 66 gegen 105, 81, 46, 34, 73 in den fünf Vorjahren. Von den einzelnen Vororten hatte Charlottenburg 521 Aerzte (1903: 508), Schöneberg 198 (179), Wilmersdorf 78 (65), Rixdorf 47 (36), Lichterfelde 38 (36), Pankow 19 (19), Steglitz 18 (20), Friedenau 16 (12), Zehlendorf 17 (15); ferner Spandau 43 (47), Köpenick 8 (6), außerdem kleinere Ortschaften 203. In Berlin und seinen Vororten wohnt also der achte Teil aller deutschen und mehr als der fünfte Teil aller preußischen Aerzte. Von den letzteren lebten in den 24 Städten mit über 100000 Einwohnern 1625 (Charlottenburg und Schöneberg sind hier mitzurechnen) $7406=40,1 \%$; in den 28 Städten mit $45-100000$ Einwohnern $1625=8,8 \%$ in den übrigen Orten Preußens $9449=51,1 \%$. Die Verteilung der Aerzte auf Großstädte, Mittelstädte, Kleinstädte ist demnach dieselbe geblieben wie im Vorjahre. Nach dem Urteil Sachkundiger wird auch in Zukunft kein erheblicherer Anteil auf die kleineren Orte kommen, da dort im allgemeinen nicht mehr Aerzte als jetzt die Möglichkeit einer Existenz finden können. Eine mehr oder weniger große Zunahme der Aerztezahl war festzustellen in den preußischen Großstädten Breslau, Köln, Frankfurt, Düsseldorf, Dortmund, eine Abnahme in Königsberg und Kiel, eine geringere in Danzig; in den übrigen 14 ist die Aerztezahl gleich oder fast gleich geblieben. Von den preußischen Mittelstädten hatte der Kurort Wiesbaden besonders viele Aerzte 237 (1903: 235), ferner die Universitätsstadt Bonn 183 (198). Die Zahl der Aerzte war zwischen 87 und 55 in Münster, Görlitz, Erfurt, Potsdam, Koblenz, Bochum, Bromberg, Frankfurt a. O., zwischen 49 und 25 in Liegnitz, Bielefeld, Rixdorf, Hagen, Osnabrück, Spandau, Beuthen, Brandenburg a. H., München-Gladbach, Flensburg, Gleiwitz, Remscheid, Mülheim a: Rhein, Elbing, Harburg, Solingen; in Königshütte endlich 17, in Linden bei Hannover 14.

Von den außerpreußischen Städten wiesen München, Leipzig, Straßburg, Nürnberg, Braunschweig ein erhebliches, Stuttgart und Chemnitz ein geringes Mehr an Aerzten, Dresden dagegen eine Verminderung auf. In der letzteren Stadt war im Vorjahre eine erhebliche Zunahme, um 57, festgestellt worden.

Von einer Berechnung des Verhältnisses der Aerztezảhl zur Anzahl der Bewohner der Städte ist in diesem Jahre Abstand genommen worden, da seit der Volkszählung von 1900 ein zu langer Zeitraum verflossen ist, als daß die damaligen Zahlen für jetzt noch annähernd als zutreffend angesehen werden könnten.

Die wirtschaftliche Lage des ärztlichen Standes erfuhr in dem abgelaufenen Jahre keine Besserung. Die Zwistigkeiten mit den Krankenkassen nahmen einen immer schärferen Charakter an und erregten besonders gelegentlich des Leipziger Aerztestreiks die Aufmerksamkeit der Regierungen, der Parlamente, der politischen Presse sowie weiter Kreise der Bevölkerung, ohne daß leider ein gangbarer Weg zu einer dauernden Verständigung gezeigt wurde. In bedauerlicher Weise wuchs die Erbitterung auf beiden Seiten; es hatte dies einen engeren und festeren Zusammenschluß der Aerzte zu gemeinsamer Abwehr zur Folge.

Daß die Ueberfüllung des ärztlichen Berufs, welche mit in erster Linie die mißliche Lage des Standes verursacht, in absehbarer Zeit sich weniger fühlbar mache, erscheint ausgeschlossen, wenn auch in den letzten Jahren die Zahl der Medizinstudierenden auf den deutschen Hochschulen sich etwas zu vermindern scheint. Nachdem im Sommersemester 1890 diese ihren Höchststand mit 8986 erreicht hatte, ging sie bis Wintersemester $1893 / 94$ auf 7882 zurück, blieb dann bis
Wintersemester 1899/1900 auf ziemlich gleicher Höhe, um dann weiter zu sinken: Wintersemester 1902/03: 7419, Sommersemester 1903: 7265, Wintersemester 1903/04: 7042, Sommersemester 1904: 6701.

Das äußere Ansehen eines Standes wird nur dann keine Einbuße erleiden, wenn derselbe nicht an innerem Werte verliert. In dieser Beziehung darf das Streben der deutschen Aerzte, sich auf der Höhe der Wissenschaft zu erhalten, als ein erfreuliches Zeichen angesehen werden. Die von dem Zentralkomitee für ärztliche Fortbildungskurse veranstalteten Vorlesungen und Uebungen erfreuen sich eines regen Besuches. Aeltere und jüngere Kollegen widmen sich dort nach allgestrengter Tagesarbeit mit erfreulichem Eifer dem Studium. So waren z. B. für die Vortragsreihe "Erste Hilfe" in Berlin nicht weniger als 400 Teilnehmer eingeschrieben. Auch die neu eingerichteten oder einzurichtenden medizinischen Akademien werden außer für Ablegung des praktischen Jahres auch der ärztlichen Fortbildung dienstbar gemacht werden. Sieht man ferner, wie überall die Aerzte ihre Zeit und Mühe in selbstloser Weise den Bestrebungen für Hebung der öffentlichen Gesundheitspflege widmen, so darf man wohl nicht ganz ohne Hoffnung für die Zukunft sein. Ein von solch idealer Lebensanschauung getragener Stand, der seine Befriedigung im Dienste der Nächstenliebe findet, der andererseits von solcher Bedeutung für das Gemeinwohl ist, muß über kurz oder lang auch wieder seine frühere soziale Stellung erlangen. 